\title{
Disclosure of congenital cleft lip and palate to Japanese patients: reported patient experiences and relationship to self-esteem
}

\author{
Tomoko Omiya ${ }^{1 *},{\text { Mikiko } \text { Ito }^{2} \text { and Yoshihiko Yamazaki }}^{3}$
}

\begin{abstract}
Background: The present study investigated when and how Japanese people with cleft lip and palate (CL/P) learn that their condition is congenital; the perceived effects of withholding the CL/P diagnosis on patients; and whether the resulting social experience and self-esteem are related. A questionnaire survey was conducted in 71 adults with $\mathrm{CL} / \mathrm{P}$ recruited through a hospital, a patients' association, and by snowball sampling.

Results: The participants became aware of their physical difference in childhood, but many reported difficulty in understanding their condition. Participants reported that their families avoided the topic of diagnosis. Participants who understood their condition during childhood rather than in adulthood were significantly more likely to consider this scenario as positive $(p<0.001)$. Although stigmatising experiences were extremely painful, most patients hid their suffering, making it more difficult to obtain social support. Participants with high self-esteem were more likely to feel that they received adequate support.

Conclusions: It is important to explain the congenital nature of CL/P sufficiently and early. In addition, openness by the family about the diagnosis, rather than avoidance, may improve patients' self-esteem. Sufficient support from family, health care providers, and significant others is needed for patients to develop adequate self-esteem.
\end{abstract}

Keywords: Cleft lip and cleft palate, Self-esteem, Self-stigma, Disclosure, Japan

\section{Background}

According to nationwide monitoring by the Japan Association of Obstetricians and Gynaecologists, the annual incidence of congenital abnormalities in new-borns is $3-5 \%$ [1]. One of the most prevalent visible morphologic abnormalities is cleft lip and palate $(\mathrm{CL} / \mathrm{P})$. Compared with the CL/P incidence of 1 in 800 in Europe and the United States, the incidence in Japan is higher at 1 in $500[2]$.

Treatment for CL/P proceeds in stages and begins 3-6 months after birth with the attending physician first discussing the situation with the parents. A therapeutic plan is introduced to the parents, and the parents and physicians come to an agreement. Team-based care is common in Japan and includes oral surgeons, plastic surgeons, otolaryngologists, orthodontists, speech therapists,

\footnotetext{
*Correspondence: toomiya-tky@umin.ac.jp

${ }^{1}$ Community Nursing, Faculty of Nursing, Toho University, 4-16-20

Omori-Nishi, Ota-ku, Tokyo 143-0015, Japan

Full list of author information is available at the end of the article
}

and others, but parents and physicians remain at the centre of treatment decisions, not just during infancy, but continuing into adulthood.

Some healthcare providers previously believed that disclosing the diagnosis might cause greater harm than good to their patients, especially children. Consequently, professional appeals to "do no harm" resulted in nondisclosure or partial disclosure of health-related information [3]. Healthcare providers believed that they should protect paediatric patients from their diagnoses and prognoses, believing that young children could not comprehend concepts such as death, serious illness, or medical treatment. A paradigm shift in medicine toward greater patient autonomy and shared decision-making led to a more open disclosure policy with children in the 1970s and 1980s. Subsequent studies have indicated that young children could achieve a basic understanding of complex medical phenomena [3].

Clarifying the explanation of $\mathrm{CL} / \mathrm{P}$ to patients and the effects of this condition is important. However, in Japan, 
virtually no study has investigated how withholding the truth or avoiding clear explanation is perceived and interpreted by people with CL/P. Walesky-Rainbow and Morris also raised these concerns in Europe and the United States, but it has not been examined further in these regions [4]. Studies of the psychosocial aspects of $\mathrm{CL} / \mathrm{P}$ in Japan have focused primarily on the process of acceptance by the child's mother [5], disclosure of the prenatal diagnosis of CL/P [5], and other factors concerning the parents, especially the mother. For the patients themselves, very few studies have been conducted on the parent-child relationship and personality traits [6].

In Europe and the United States, numerous studies have examined psychosocial factors in patients with CL/P and report that self-perception [7], self-esteem [8], and adjustment [9] are considered important indicators of psychosocial health in these patients. In particular, adolescents often face severe stigma, such as teasing or bullying, and low self-esteem and self-concept have been reported [10]. With respect to Japanese cultural differences, Hirose suggested that the Japanese characteristic of avoiding direct discussion of congenital facial defects has prevented the accumulation of research [11]. There are cultural factors in Japan that may underlie the unclear explanations and truth avoidance. Truth-telling means not hiding the truth and not telling a lie; it means explaining the entire treatment process rather than just naming the condition. Japanese culture includes a descriptive style that encourages ambiguity in speech and avoidance of clarity, and a culture of shame and ridicule related to facial appearance. Japan is a racially homogenous nation with a culture that respects harmony and places great value on sameness. This results in extreme sensitivity to differences, and differences in facial appearance readily lead to exclusion. People fear that they too will be excluded if they discuss CL/P openly. Because surgical treatment helps decrease the visible appearance of CL/P, people in Japan choose to believe the condition never existed. Finally, Japanese culture includes a concept called sekentei, which concerns public image and respectability. Senkentei favours avoidance of shameful behaviour, and promotes concern over public perception, fear of social criticism, selfrestraint in deference to others, and privacy within the family [12].

In the present study, we used a questionnaire survey to clarify when and how people with $\mathrm{CL} / \mathrm{P}$ became aware of their condition, their understanding of the condition, and the perceived effects of withholding the truth. Our objective was to examine the relationships between the timing of their awareness/disclosure of $\mathrm{CL} / \mathrm{P}$, their relationships with family and physicians, and self-esteem.

\section{Methods}

This study was approved by the Ethics Committee of the Graduate School of Medicine and Faculty of Medicine, The University of Tokyo (Approval number 1277).

\section{Participants}

There were three inclusion criteria for patients: (1) adult $\geq 20$ years old; (2) no physical complications or comorbidities (exclusion criteria: intellectual delay or disability, physical disability, and congenital disease such as Pierre Robin syndrome); and (3) knowledge of the name and nature of the diagnosis (i.e., aware of the congenital nature). Criterion (3) was particularly carefully verified in participants. All participants gave their informed consent after receiving a written explanation of the purpose and content of the study. Knowledge of the CL/P diagnosis was verified based on answers to the following questions: Question 1: Your concern during the hospital examination was your lips, but what specifically did you have questions about? If the subject did not include, cleft lip and palate in their answer, they were not enrolled in the study. Question 2: What type of illness do you believe affects your mouth, or what do you believe the underlying cause is? Subjects were enrolled if they stated that they were born with the condition, the lip was split from birth, or the lip had never fused, indicating some understanding of the congenital nature of CL/P.

\section{Subject recruitment}

Participants were recruited by three routes as follows:

A) Hospital route: Subjects learned about the study from physicians at the Osaka University Dental Hospital in the CL/P specialty service and were selected by an attending physician during outpatient examination. The physicians were given the following guidelines for subject participation: 1) exclusion of patients immediately pre- and postoperatively; 2) inclusion of subjects felt to be mentally stable; and 3) subjects who appeared to understand the meaning of cleft lip and palate based on the two questions described earlier, as evaluated by the chief physician.

B) Snowball sampling: Subjects were acquainted with the researchers or other subjects, such as during hospitalisation or outpatient visits, on the internet, or at off-line meetings. The sample included four subjects known by the author, two direct acquaintances of the researcher, and two friends of the researcher's relatives.

C) Patient association: The Association of Cleft Lip and Cleft Palate in Japan, one of the largest support groups for parents of children with $\mathrm{CL} / \mathrm{P}$, has a "cleft lip friend's meeting," which is held for patients 
with CL/P and an accompanying adult. The director of the group agreed to cooperate, and a questionnaire was distributed to the membership (subject with an accompanying adult).

From August 2005 to November 2010, questionnaires were distributed to 11 participants by the hospital route, 36 participants by the snowball sampling route, and 52 participants through the patient association. Ten of 11 participants (90.9\%) from the hospital route, 35 of 36 participants (97.2\%) from snowball sampling, and 27 of 52 participants (51.9\%) from patient meetings returned the questionnaires. One subject initially enrolled through the snowball sampling was 18 years old and thus did not meet the Japanese adult age cut-off criterion of $\geq 20$ years; the patient was excluded. The patient attributes and characteristics are shown in Table 1.

\section{Survey and measurements}

A Cronbach coefficient alpha was calculated for each survey for the 71 included participants.

\section{Table 1 Participant sociodemographic characteristics}

\begin{tabular}{|c|c|c|}
\hline & \multicolumn{2}{|l|}{ Total } \\
\hline & No. patients $(n=71)$ & $(\%)$ \\
\hline Sex & 20 & $(28.2)$ \\
\hline Male & 51 & $(71.8)$ \\
\hline \multicolumn{3}{|l|}{ Female } \\
\hline Age, mean \pm SD (range, $20-75$ years) & $33.8 \pm 7.5$ & \\
\hline \multicolumn{3}{|l|}{ CL/P type } \\
\hline Unilateral CL/P & 33 & $(46.5)$ \\
\hline Bilateral CL/P & 19 & $(26.8)$ \\
\hline Unknown CL/P & 8 & $(15.4)$ \\
\hline Cleft lip (unilateral) & 5 & $(7.0)$ \\
\hline Cleft lip (unknown) & 1 & $(1.4)$ \\
\hline Cleft palate & 1 & $(1.4)$ \\
\hline No answer & 4 & (5.6) \\
\hline \multicolumn{3}{|l|}{${ }^{a}$ Recruitment } \\
\hline Hospital & 10 & $(14.1)$ \\
\hline Patient association & 27 & $(38.0)$ \\
\hline Snowball sampling & 34 & $(47.9)$ \\
\hline bSelf-esteem score (range, 10-50) & $33.0 \pm 4.1$ & \\
\hline Self-stigma score (range, 3-12) & $6.29 \pm 1.8$ & \\
\hline Disclosure score (range, 3-12) & $10.7 \pm 6.2$ & \\
\hline
\end{tabular}

$\mathrm{CL} / \mathrm{P}$, Cleft lip and palate; SD, standard deviation; No., number.

${ }^{a}$ Study participants were recruited as follows: during routine examination (hospital route), through the Association of Cleft Lip and Cleft Palate in Japan (patient association route), or by acquaintance with an investigator or other participant (Snowball sampling).

${ }^{\mathrm{b}}$ Data presented as the mean \pm standard deviation.
(1) Self-esteem was measured using Rosenberg's scale [13] translated into Japanese by Yamamoto et al. [14]. This scale comprises 10 questions with five possible responses. Scoring differs from the original Rosenberg scale (10 items on a 4-point scale), but the Japanese translation is one of the most commonly used self-esteem scales in Japan [15,16]. The higher the score, the higher the self-esteem (Cronbach coefficient alpha, 0.89).

(2) Self-stigma was measured based on the self-stigma scale described by Link et al. [17]. Three questions with four possible responses reflecting the study goals were asked. Higher scores represented greater self-stigma (Cronbach coefficient alpha, 0.70).

(3) Each subject's experience of understanding the diagnosis at different time periods was evaluated as follows: elementary school, $6-11$ years of age; junior high school, 12-15 years of age; and high school, 16-18 years of age. Elementary school was further divided into early (6-8 years of age) and late (9-11 years of age) elementary stages. Subjects were asked when and how they learned about CL/P and what explanations they received.

(4) Each subject's first awareness and experience of $\mathrm{CL} / \mathrm{P}$ at different time periods was evaluated as follows: a) four questions on the concerns and understanding of the diagnosis; b) two questions on the physician-patient relationship; c) one question on family conversations about the diagnosis; d) six questions on stigmatising experiences, such as bullying, and the response to these experiences; and g) two questions on the parent-child relationship.

(5) Based on a previous survey [18], the degree of disclosure to others about $\mathrm{CL} / \mathrm{P}$ was determined using an original survey with three questions and four possible responses that considered the relationship between disclosure and self-stigma. The three questions were: "Can you talk about your condition being congenital?"; "Can you state specifically that it is a congenital anomaly?"; and "Even when not asked, can you talk about your condition when necessary?" The higher the score, the greater the subject's tendency to disclose his or her CL/P condition to others (Cronbach coefficient alpha, 0.80).

(6) Eight questions measured demographic characteristics as follows: age, sex, educational background, marital status, employment status, and socioeconomic group.

\section{Statistical analysis}

Evaluation of patients' understanding of the CL/P diagnosis by time period

The t-test was used for continuous data, and the chisquared test was used for cross tabulation. Multiple 
comparisons (Kruskal-Wallis and Bonferroni correction) were performed for the 71 participants to evaluate their understanding of $\mathrm{CL} / \mathrm{P}$ at different time periods (before and during elementary school, junior high school, and high school). The data were also subjected to regression analysis with self-esteem category as the dependant variable.

\section{Self-esteem categorisation}

Participants were divided into high, middle, and low groups by self-esteem score and comparatively analysed, a technique employed in numerous fields [16]. Typically, when dividing participants into groups, a cut-off of the mean \pm 1.5 standard deviation (SD) is sometimes used or participants are divided into three equal-sized groups [19]. Because the self-esteem scores in this study were higher than those in other Japanese studies of the same age groups [20], a cut-off point of the mean $\pm 1.5 \mathrm{SD}$ and dividing the participants into three equal groups were considered inappropriate. After considering the mean and median values, the distributions reported in previous studies, and the interview results, we divided the participants into three groups based on the self-esteem scores as follows: high self-esteem group, total score $\geq 36$ points ( 25 participants); middle self-esteem group, $29<$ total score $<$ 36 (26 participants); and; low self-esteem group, total score $\leq 29$ points (20 participants). The group allocations were based on a previously published protocol [18].

\section{Results}

The sociodemographic characteristics of the participants are shown in Table 1 . The majority of participants (71.8\%) were women, and the mean age of the group was 33.8 years. Unilateral $\mathrm{CL} / \mathrm{P}$ was the most common diagnosis (46.5\%), but some participants were unsure of whether their $\mathrm{CL} / \mathrm{P}$ was complete or incomplete, or unilateral or bilateral.

\section{Age of $\mathrm{CL} / \mathrm{P}$ disclosure and patient response}

As shown in Table 2, 67 participants (94\%) noticed that their nose and mouth were different from those in others during pre-elementary or elementary school. Upon realising the difference, 38 (53.5\%) asked another person why. Most participants asked their parents, and $28(39.4 \%)$ were told that the condition was congenital, but $17(24.0 \%)$ were given untrue explanations, such as an injury resulting from a fall out of bed or outside, or injury due to the umbilical cord being wrapped around the head.

\section{Frequency of discussion on $\mathrm{CL} / \mathrm{P}$ with others and underlying motivations}

Among the participants who noticed that they had CL/P, $28(39.4 \%)$ did not ask anyone about their condition. In
Table 2 Participants knowledge of their cleft lip and

palate $(\mathbf{n}=71)$

n $(\%)$

Age when participants noticed their mouth/lip was different

Pre-elementary school (0-5 years)

$30 \quad(42.3)$

Early elementary school (6-8 years)

29 (40.8)

Late elementary school (9-11 years)

$8 \quad(11.3)$

Junior high school (12-15 years)

$4 \quad(5.6)$

Action upon noticing their CL/P

Asked someone

Did not ask anyone

Other response/No answer

Explanation provided by others upon asking (multiple answers)

Condition name

Identified as a malformation

Congenital etiology

Cause

False explanation

Age when participants learned the name, cause, and congenital name of malformation

Pre-elementary school

Early elementary school

Late elementary school

Junior high school

High school

Post-high school ( $\geq 19$ years)

${ }^{a}$ Manner that participants obtained the above information $(n=70)$

Told by someone

Learned on own

Learned by chance

$\mathrm{CL} / \mathrm{P}$, cleft lip and palate.

aparticipants learned of their own CL/P from the following individuals: mother $(n=24)$, attending physician $(n=3)$, other physician $(n=2)$, father $(n=2)$, friend $(n=2)$, or other $(n=7)$.

the free description column of the questionnaire, nine participants stated that they felt the topic was taboo based on their parents' demeanour and on daily exchange with family members. One patient stated that "it was hopeless even to ask about that face," (4) and "that my parents would feel sad if I asked about my diagnosis" (4).

\section{Manner of CL/P disclosure and associated self-esteem}

Thirty-four (47.8\%) participants knew that their condition was congenital by the end of elementary school (11 years of age), while 22 (30.1\%) knew after age 19 years. Twentyfour participants learned about their $\mathrm{CL} / \mathrm{P}$ from their mothers; three from the attending physician; and two from their father. However, several participants reported learning about their $\mathrm{CL} / \mathrm{P}$ in an undesirable and traumatic 
way. For example, one participant became aware during the birth of her child; the obstetrician diagnosed her child with $\mathrm{CL} / \mathrm{P}$ and informed the mother of her own $\mathrm{CL} / \mathrm{P}$ simultaneously. A total of $22(31 \%)$ participants learned about $\mathrm{CL} / \mathrm{P}$ on their own using books and the Internet, and 19 participants learned after 19 years of age, indicating that many participants were unaware of their condition for a long period.

As shown in Table 3, the multiple comparisons (KruskalWallis, Bonferroni correction) revealed that participants who learned about their $\mathrm{CL} / \mathrm{P}$ while in elementary school (6-11 years of age; mean score, $3.2 \pm 0.82$ ) were more likely to believe that it was the best time than were participants who learned about it after high school. Two participants wrote that "Being told too early is not good," and one replied that "The time did not matter," but most participants did not consider themselves too young to understand $\mathrm{CL} / \mathrm{P}$ during kindergarten or elementary school. The mean scores for participants who were first informed during junior and senior high school were significantly lower at $2.82 \pm 0.41$ $(\mathrm{p}<0.001)$ and $1.92 \pm 0.63$, respectively.

Table 4 shows the participants' experiences during each time period. Even during high school (16-18 years of age), approximately $38 \%$ did not understand that their facial scarring and articulation disorders were due to CL/P. Similarly, only $48 \%$ of participants who visited a hospital or were hospitalised during high school understood the reason and the nature of the treatment.

\section{Family and physician relationships}

As shown in Table 4, after reaching adulthood, only 32.9\% of participants asked their doctors about CL/P, and less than $40 \%$ felt that they currently had good communication with their doctors. Only 17 patients (23.9\%) felt that their doctors understood their feelings and goals after age 19 years, and 29 patients (40.8\%) answered no to the same question.

In general, the participants enjoyed a good relationship with their parents as children, with $84.5 \%$ (60) reporting

Table 3 Participants' initial awareness of and response to $\mathrm{CL} / \mathrm{P}$ according to time period

\begin{tabular}{|c|c|c|c|}
\hline $\begin{array}{l}\text { Period when participant learned } \\
\text { about } \mathrm{CL} / \mathrm{P}\end{array}$ & $\mathbf{n}$ & $\begin{array}{l}\text { Satisfaction } \\
\text { score }^{a}\end{array}$ & p-value ${ }^{b}$ \\
\hline Before or during elementary school & 34 & $3.24 \pm 0.8$ & $0.74^{*}$ \\
\hline Junior high school & 11 & $2.82 \pm 0.4$ & $0.01^{* *}$ \\
\hline High school/post-high school & 26 & $1.92 \pm 0.6$ & $0.000^{* * *}$ \\
\hline \multicolumn{4}{|c|}{$\begin{array}{l}\text { CL/P, cleft lip and palate. } \\
\text { a Participants were asked to respond to the following statement: "I am satisfied } \\
\text { that I came to know about my } \mathrm{CL} / \mathrm{P} \text { condition during this time period," with } \\
\text { one of four responses (strongly agree [4 points] to strongly disagree [1 point]). } \\
\text { A higher score indicated greater satisfaction by the respondent. } \\
\text { b Significance defined as } \mathrm{p}<0.05 \text { and determined by the Kruskal-Wallis test as } \\
\text { follows: }{ }^{*} \text { comparing elementary and junior high school periods, }{ }^{* *} \text { comparing } \\
\text { junior and high school/post-high school periods, and }{ }^{* * *} \text { comparing } \\
\text { elementary school and high school/post-high school periods. }\end{array}$} \\
\hline
\end{tabular}

a good or somewhat good relationship, and trust with their parents. However, the patients also reported a tendency to avoid frank discussion of CL/P within the family or openly discuss problems and concerns related to the diagnosis. Overall, 37 (52.1\%) of participants reported being raised not to be self-conscious of their CL/P.

As shown in Table 4, a high percentage of patients reported experiencing stigma due to teasing during late elementary school, which peaked during junior high school. Most participants discussed their anxieties with their mothers, but more than 29 participants (40\%) experiencing stigma during early elementary school did not discuss the problem with anyone. Several participants who did seek outside counsel stated that the support was insufficient. As participants matured, more people were available to provide advice, but $60-80 \%$ of subjects still did not discuss the matter with anyone else. Among the reported reasons, participants expressed resignation that the parents would be able to intervene or would dismiss their concerns. Participants also wished to avoid saddening their parents over their experienced of being bullied.

\section{Comparison between self-esteem groups}

No significant differences were observed between the selfesteem groups in participant characteristics, the parentchild relationship, the incidence of teasing, and social stigma. However, as shown in Table 5, the high self-esteem group showed significantly lower self-stigma and significantly greater disclosure of $\mathrm{CL} / \mathrm{P}$ to others than the other groups. In addition, during periods of social difficulty, for example when being bullied in elementary school, individuals with high self-esteem were more likely to receive sufficient support from someone important to them. The regression analysis was also performed using self-esteem as the dependent variable; however, there were no statistically significant differences between the participant groups.

\section{Discussion}

\section{Participant characteristics}

Ours is one of the few studies assessing the disclosure to and understanding of people with the congenital condition $\mathrm{CL} / \mathrm{P}$. In Japan, the psychosocial study of $\mathrm{CL} / \mathrm{P}$ is extremely sensitive. Even in a hospital setting, when adult subjects attended with a parent, several parents secretly refused to allow participation because they had not yet fully explained CL/P to their son or daughter (aged 20 and older). In Japan, it is not unusual for parents to accompany their adult children to medical appointments, even those older than 20 years of age. Parents often feel guilt over the congenital nature of $\mathrm{CL} / \mathrm{P}$ and also wish to follow the long-term progress. The parents' permission was required by the chief physician overseeing the study if the parent was present with their child, even if the child was older than 20 years of age and mentally able to consent. 
Table 4 Participants' answers to questions about having CL/P by time period $(n=71)^{a}$

Early elementary Late elementary Junior high

High school $\geq 19$ years Never school (6-8 years) school (9-11 years) school (12-15 years) (16-18 years)

n $\%$

$\%$ n

n $\%$

n $\%$

n $\%$

$\bar{n} \%$ n

\section{Awareness and understanding of CL/P}

I was worried about the scars around my mouth and the shape of my nose.

I was worried about my speech.

I understood that the scars around my mouth and my speech were due to a medical condition.

I understood the reason for the hospital visits and admissions.

Relationship with physician

I actively asked my doctor questions.

I think my doctor knows my feelings and hopes well.

Family conversations about CL/P

It was natural to talk about my medical condition with my family.

\begin{tabular}{llllllllllll}
35 & $(50.0)$ & 43 & $(61.4)$ & 47 & $(67.1)$ & 50 & $(71.4)$ & 46 & $(65.7)$ & 1 & $(1.4)$ \\
26 & $(36.6)$ & 25 & $(35.2)$ & 32 & $(45.1)$ & 28 & $(39.4)$ & 24 & $(33.8)$ & 19 & $(26.8)$ \\
22 & $(31.9)$ & 33 & $(47.8)$ & 43 & $(62.3)$ & 43 & $(62.3)$ & 50 & $(72.5)$ & 2 & $(2.9)$ \\
13 & $(18.3)$ & 20 & $(28.2)$ & 35 & $(49.3)$ & 37 & $(52.1)$ & 42 & $(59.2)$ & 7 & $(9.9)$ \\
& & & & & & & & & & & \\
0 & $(0.0)$ & 2 & $(2.9)$ & 2 & $(2.9)$ & 11 & $(15.7)$ & 23 & $(32.9)$ & 23 & $(32.9)$ \\
6 & $(8.5)$ & 5 & $(7.0)$ & 7 & $(9.9)$ & 16 & $(22.5)$ & 17 & $(23.9)$ & 29 & $(40.8)$ \\
& & & & & & & & & & & \\
11 & $(16.7)$ & 17 & $(25.8)$ & 17 & $(25.8)$ & 18 & $(27.3)$ & 23 & $(34.8)$ & 24 & $(36.4)$ \\
& & & & & & & & & & & \\
46 & $(64.8)$ & 52 & $(73.2)$ & 45 & $(63.4)$ & 27 & $(38.0)$ & 23 & $(32.4)$ & 2 & $(2.8)$ \\
16 & $(34.8)$ & 21 & $(40.4)$ & 27 & $(60.0)$ & 9 & $(33.3)$ & 6 & $(26.1)$ & - & - \\
33 & $(47.1)$ & 44 & $(62.9)$ & 30 & $(42.9)$ & 8 & $(11.4)$ & 6 & $(8.6)$ & 17 & $(24.3)$ \\
13 & $(39.4)$ & 21 & $(47.7)$ & 19 & $(63.3)$ & 0 & $(0.0)$ & 1 & $(16.7)$ & - & - \\
25 & $(35.2)$ & 35 & $(49.3)$ & 33 & $(46.5)$ & 10 & $(14.1)$ & 6 & $(8.5)$ & 24 & $(33.8)$ \\
14 & $(56.0)$ & 18 & $(51.4)$ & 21 & $(63.6)$ & 3 & $(30.0)$ & 1 & $(16.7)$ & - & - \\
\hline
\end{tabular}

Stigmatizing experiences

Someone pointed out my face or speech.

It was hard to have my face or speech pointed out.'

Someone made fun of my face or speech.

It was hard to be mocked about my face or speech.b

Someone used abusive words or violence.

It was hard to experience abusive words or violence. ${ }^{b}$

Participants were asked to circle all periods when the answer was yes. The number of participants circling each item was calculated for each period.

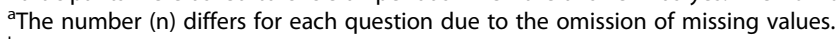

bercentages were calculated based on the total population of people with stigmatizing experiences. 
Table 5 Significant differences in survey scores and responses between the high and low self-esteem groups

\begin{tabular}{|c|c|c|c|}
\hline & $\begin{array}{l}\text { High SE group } \\
(n=25)\end{array}$ & $\begin{array}{l}\text { Low SE group } \\
(\mathrm{n}=20)\end{array}$ & \\
\hline & Mean \pm SD & Mean \pm SD & p-value \\
\hline Self-esteem score (range, 10-50) & $41.0 \pm 3.8$ & $22.5 \pm 4.8$ & 0.000 \\
\hline Self-stigma score (range, 3-12) & $5.0 \pm 0.9$ & $7.7 \pm 2.8$ & 0.006 \\
\hline Disclosure of $\mathrm{CL} / \mathrm{P}$ to others (range, 3-12) & $12.1 \pm 1.4$ & $7.0 \pm 3.0$ & 0.000 \\
\hline I was raised not to be conscious about my $\mathrm{CL} / \mathrm{P}^{\mathrm{a}}$ & $12(48.0 \%)$ & $16(80.0 \%)$ & 0.045 \\
\hline $\begin{array}{l}\text { Period during which participants received sufficient support from someone important to them } \\
(\text { range, } 1-5)^{b, c}\end{array}$ & $1.8 \pm 0.8$ & $1.0 \pm 0.7$ & 0.054 \\
\hline Period during which participant actively asked doctors questions about $C L / P(\text { range, } 1-5)^{\mathrm{b}, \mathrm{c}}$ & $1.2 \pm 1.0$ & $0.6 \pm 0.4$ & 0.079 \\
\hline
\end{tabular}

SE, self-esteem; SD, standard deviation; CL/P cleft lip and palate.

a Number (percentage) of participants who answered "yes" on this survey question.

${ }^{\mathrm{b}}$ Responses from 45 participants (25 high SE and 20 low SE subjects) who consulted someone upon experiencing difficulty with their CL/P.

'For each period (early elementary school, 6-8 years; late elementary school, 9-11 years; junior high school, 12-15 years; high school, 16-18 years; and $\geq 19$ years),

1 point was given if the subject answered that he/she was treated well, for a maximum of 5 points.

${ }^{\mathrm{d}}$ Significance defined as $\mathrm{p}<0.08$.

There were also several cases in which the attending physician could not determine whether the subject understood the nature of his or her condition.

Compared with the total population of patients with $\mathrm{CL} / \mathrm{P}$ in Japan, 71 participants is certainly not large, which is a limitation of this study. However, considering that at least 20 parents declined to allow their children to participate in the study to protect them, collecting detailed data from even 71 Japanese participants is quite exceptional.

\section{Manner of disclosure and family conversations}

It appears that participants did not receive sufficient explanation when they asked their parent(s) about their CL/P. Many participants did not fully understand their condition until entering adulthood. The reasons underlying this inadequate explanation from the parents may include anticipation and anxiety about potential discrimination that their child may experience, such as bullying and stigmatisation. We speculate that the expectation of parents that they can deny the existence of CL/P after surgical repair favours hiding the condition as a strategy of coping with social stigma [21]. The parents hide the details of the condition not only from surrounding people but also from their children, likely out of concern. They fear that their child may be hurt from knowing the truth, or they fear that their child may offend others due to the congenital diagnosis. Currently, the manner in which subjects are told about their diagnosis depends not on physicians but on parents, and many parents fail to disclose the truth for a long time [22]. This may reflect the lack of support on how to explain the condition to their children; therefore, children grow up without understanding $\mathrm{CL} / \mathrm{P}$.

Notably, the participants in this study desired a sufficient explanation at an earlier stage. In the United States, the idea that even a child has the basic human right to full knowledge of his or her medical condition is taken for granted. For example, in a study of children with leukaemia, investigators found that disclosing information such as the name of the disease and the treatment plan relieved anxiety in the children, increased their trust in healthcare providers, and enhanced the ability to self-control the disease [23]. Physicians play a role in truth-telling with patients with CL/P, but Saiki-Craighill found that many doctors were generally more conservative in this respect than parents, even though Japan adheres to the United Nations Declaration of the Rights of the Child, as does the United States [24]. The resistance of physicians to discussions about the diagnosis may be an important factor in patients' awareness and acceptance of their diagnosis. For people with $\mathrm{CL} / \mathrm{P}$, who require prolonged treatment, understanding their current status and the necessity for treatment are important. One report in Japan observed that many people with $\mathrm{CL} / \mathrm{P}$ discontinue corrective treatment, indicating that explanation of medical support to people with $\mathrm{CL} / \mathrm{P}$ may be necessary to prevent dropout [25]. According to a survey conducted in Japan on doctor-patient communication, patients with depression $(\mathrm{n}=2020)$ reported that $55.0 \%$ of their interactions were satisfactory, whereas $14.3 \%$ were unsatisfactory [26]. Because $\mathrm{CL} / \mathrm{P}$ and depression are different, a direct comparison between these studies is difficult. However, in cases of $\mathrm{CL} / \mathrm{P}$, it appears that the communication between patients and doctors is insufficient.

\section{Avoidance of $\mathrm{CL} / \mathrm{P}$ discussion in the family and its impact and importance}

In our study, approximately $52 \%$ of participants reported being raised not to be self-conscious of their CL/P. However, regardless of whether their avoidance behaviour stems from care or affection for the child, when parents insist on pretending that the $\mathrm{CL} / \mathrm{P}$ does not exist, talking about the diagnosis may become taboo in the family. When the child recognises a potential difference in the 
facial appearance compared with others, he or she may be unable to discuss the matter with family and may not seek support. The majority of participants experienced the greatest amount of teasing related to their CL/P during junior high school, but many did not consult anyone. One reason reported by the participants was the resignation that seeking help would be ineffective and would worry their parents. Scambler \& Hopkins reported that when suffering is hidden, stigma is internalised, and self-stigma may result [27]. Similarly, Matsumoto et al. stated that when suffering is hidden, individuals have no psychological strength to face their CL/P [28]. Approximately $80 \%$ of participants in the low self-esteem group reported being raised not to be self-conscious of their CL/P compared with $48 \%$ in the high self-esteem group; yet, self-stigma was significantly higher in the low selfesteem group than in the high self-esteem group. These results support those reported by both Scambler et al. and Matsumoto et al. $[27,28]$.

\section{Clinical implications}

Our results suggest that people with $\mathrm{CL} / \mathrm{P}$ routinely have their facial difference pointed out repeatedly over a long period beginning at infancy. They experience stigma and feel uncertainty over lacking full knowledge about their diagnosis. This situation may seem trivial, but it slowly degrades affected individuals, deprives the child of selfesteem and self-affirmation, and can lead to appreciable psychological trauma. Ensuring safety and security is vital in the initial treatment of psychological trauma [29]; therefore, it is important that people with $\mathrm{CL} / \mathrm{P}$ receive warm support from their family and significant others, and that they find a safe place to relax. Miyaji stated that those who have no choice but to recognise their difference find safety and security with their peers [29]. Therefore, interaction with other patients with $\mathrm{CL} / \mathrm{P}$ is considered very important, and the patient's medical team should provide this emotional support. Additionally, the medical team plays a crucial role in explaining the diagnosis to subjects and promoting their understanding of $\mathrm{CL} / \mathrm{P}$, as well as providing psychological support and intervention. Omiya et al. reported that explanations must be tailored to the patient's level of understanding, especially concerning age [18]. Multiple explanations throughout a patient's lifetime are necessary, and the patient-care team must choose the best person(s) to initiate and continue those discussions. More research is needed on the training of both medical personnel and parents to better communicate the nature of $\mathrm{CL} / \mathrm{P}$ to patients and to overcome the cultural barriers currently existing in Japan.

\section{Study limitations and challenges}

This study is biased in its study population, which was carefully selected by attending physicians who assessed the subjects' psychological status and treatment history. The study participants had higher-than-average selfesteem scores compared with the scores in an earlier study [30]. This suggests that the participants in our study were in a better psychological status, and therefore, subjects who were suffering from more serious psychosocial problems may have been underrepresented. In Japan, the incidence of $\mathrm{CL} / \mathrm{P}$ is slightly higher in men than in women, but $71.8 \%$ of our subjects were female and $28.2 \%$ were male. The difference between our ratio and the prevalence could influence the results; therefore, caution is warranted when generalising the implications.

Because the subjects were surveyed retrospectively, with some experiences dating back several decades, ideally, parents and healthcare providers should also have been surveyed to improve the credibility of the study, understand the complete clinical picture, and obtain a multifaceted view. With the medical advances since the time of surgery in the subjects, it may currently be easier for parents to explain the condition.

Finally, our study was specific to the Japanese CL/P population and may not be applicable in other countries.

\section{Conclusion}

Our participants reported long-standing difficulty with understanding their condition. Their stigmatising experience rates were high, particularly during junior high school age. Although the stigmatising experiences were extremely painful, many patients hid their suffering, making it more difficult to obtain social support. The participants felt that their families avoided the topic of their diagnosis. Within the family, talking about the condition, rather than considering it taboo, would likely make it easier for patients with $\mathrm{CL} / \mathrm{P}$ to discuss their troubles and worries. Participants with high self-esteem felt that they received adequate support and were open with others regarding their congenital condition. It is important to explain the congenital nature of $\mathrm{CL} / \mathrm{P}$ sufficiently and early. Sufficient support from family, health care providers, and significant others is necessary for patients to develop adequate self-esteem.

\section{Competing interests}

The authors declare that they have no competing interests.

\section{Authors' contributions}

TM designed the study, collected and analysed the data, and drafted the manuscript. Ml and YY helped design the study and draft the manuscript. All authors read and approved the final manuscript.

\section{Acknowledgements}

We thank all participants for their time and effort, especially Ichiro Kojima, Tatsuhiko Miya, and Professor Mikihiko Kogo for collecting the data. This study received financial aid from FY2009 Grants-in-Aid for Scientific Research (Basic Research A, 21243033). 


\section{Author details}

${ }^{1}$ Community Nursing, Faculty of Nursing, Toho University, 4-16-20 Omori-Nishi, Ota-ku, Tokyo 143-0015, Japan. '2Division of Health Promotion Science, Nursing Science, Course of Health Science, Graduate School of Medicine, Osaka University, 1-7 Yamada-oka, Suita, Osaka 565-0871, Japan. ${ }^{3}$ Department of Social Welfare, Faculty of Social Welfare, Nihon Fukushi University, Okuda, Mihama-cho, Chita-gun, Aichi 470-3295, Japan.

Received: 10 September 2013 Accepted: 12 December 2014 Published: 16 December 2014

\section{References}

1. Hirahara F: Senten-ijyou monitaringu: Wagakuni to sekai no torikumi [Birth Defects Monitoring: Efforts of Japan and the World] (in Japanese). Acta Obstetrica et Gynaecologica Japonica 2007, 59:244.

2. Natsume N, Kawai T, Kohama G, Teshima T, Kochi S, Ohashi Y, Enomoto S, Ishii M, Shigematsu T, Nakano Y, Matsuya T, Kogo M, Yoshimura Y, Ohishi M, Nakamura N, Katsuki T, Goto M, Shimizu M, Yanagisawa S, Mimura T, Sunakawa H: Incidence of cleft lip or palate in 303738 Japanese babies born between 1994 and 1995. Br J Oral Maxillofac Surg 2000, 38:605-607.

3. Tse CY, Chong A, Fok SY: Breaking bad news: a Chinese perspective. Palliat Med 2003, 17:339-343.

4. Waleskyrainbow PA, Morris HL: Assessment of informative-counselling procedures for cleft-palate children. Cleft Palate J 1978, 15:20-29.

5. Nakanii M, Shinohara H, Moriguchi T: Research on the actual state of disclosure in the prenatal notification of cleft lip and palate and recommendations for a desirable support system (in Japanese). Kawasaki Medical Welfare Journal 2005, 15:103-116.

6. Kitamura Y, Ueda R, Tamaki A, Kawada S, Fujita H: Self-esteem and its related variables in children with cleft lips and/or cleft palates. Bulletin of Research Institute, National Rehabilitation Center for Persons with Disabilities 2005, 5:1-5.

7. Millard T, Richman LC: Different cleft conditions, facial appearance, and speech: relationship to psychological variables. Cleft Palate Craniofac $J$ 2001, 38:68-75.

8. Gussy M, Kilpatrick N: The self-concept of adolescents with cleft lip and palate: a pilot study using a multidimensional/hierarchical measurement instrument. Int J Pediatr Dent 2006, 16:335-341.

9. Berger ZE, Dalton LJ: Coping with a cleft II: factors associated with psychosocial adjustment of adolescents with a cleft lip and palate and their parents. Cleft Palate Craniofac J 2011, 48:82-90.

10. Strauss RP, Ramsey BL, Edwards TC, Topolski TD, Kapp-Simon KA, Thomas CR, Fenson C, Patrick DL: Stigma experiences in youth with facial differences: a multi-site study of adolescents and their mothers. Orthod Craniofac Res 2007, 10:96-103.

11. Hirose T: A literature review of psychosocial problems of children with cleft lip and cleft palate. Journal of Japanese Cleft Palate Association 1999 24:348-357.

12. Inoue T: Sekentei no Kozo [the structure of sekentei] (in Japanese). 1st edition. Tokyo: Kodansha; 2007:97-188.

13. Rosenberg M: Society and the Adolescent Self-Image. Princeton, New Jersey: Princeton University Press; 1965.

14. Yamamoto $M$, Matsui $Y$, Yamanari $Y$ : The structure of perceived aspects of self. Jpn J Educ Psychol 1982, 30:64-68.

15. Kaketa K, Nozaki T: Evaluation of structured group encounter in training for teachers with ten years of experience at an academic seminar. Journal of Hokkaido University of Education 2008, 58:175-184.

16. Matsuura N: Empirical research on relationship between inattention hyperactivity, Impulsivity, and self-esteem in inmates of a correctional facility (in Japanese). Human Developmental Research 2008, 22:291-292.

17. Link BG, Struening E, Cullen FT, Shrout PE, Dohrenwend BP: A modified labelling theory approach to mental-disorders - an empirical-assessment. Am Sociol Rev 1989, 54:400-423.

18. Omiya $T$, Ito $M$, Yamazaki $Y$ : The process leading to affirmation of life with cleft lip and cleft palate: the importance of acquiring coherence. Jpn J Nurs Sci 2012, 9:127-135.

19. Squires J, Bricker D, Potter L: Revision of a parent-completed developmen screening tool: ages and stages questionnaires. J Pediatr Psychol 1997, 22:313-328
20. Menjyu N: Analysis of the medical sociological several problems a mother of a granny birth has (in Japanese). Bulletin of International Buddhist University 2009, 47:245-261.

21. Conrad MM, Pacquiao DF: Manifestation, attribution, and coping with depression among Asian Indians from the perspectives of health care practitioners. J Transcult Nurs 2005, 16:32-40.

22. Sado A, Ishii M, Ishii Y, Moriyama T, Morita K, Gunji A, Imaizumi F, Murase K, Takahashi Y, Enomoto S: Clinical study on telling patients with cleft lip and cleft palate the name of their disease (in Japanese). Journal of Japanese Cleft Palate Association 2001, 26:97-113.

23. Leikin SL: An ethical issue in pediatric cancer care: nondisclosure of a fatal prognosis. Pediatr Ann 1981, 10:37-41.

24. Saiki-Craighill S: The attitude of pediatric oncologists regarding disclosing the nature of the illness to children. J Child Health 1998, 57:590-597.

25. Ito K, Matsuura S, Ohta K: A survey on cleft lip and cleft palate patients who discontinued corrective treatment at Orthodontic Dentistry of Hiroshima University Dental Hospital. Journal of Japanese Cleft Palate Association 1993, 18:291-299.

26. Sawada N, Uchida H, Watanabe K, Kikuchi T, Suzuki T, Kashima H, Mimura M: How successful are physicians in eliciting the truth from their patients? A large-scale internet survey from patients' perspectives. J Clin Psychiat 2012, 73:311-317.

27. Scambler $G$, Hopkins A: Being epileptic-coming to terms with stigma. Sociol Health IIIn 1986, 8:26-43.

28. Matsumoto M: Youbou-no Jko Jyuyou-Koshin/Kogairetu no Baai (Self-acceptance of looks-in case of cleft lips and cleft palate). Gendai Bunmei Kenkyu 1999, 2:88-106.

29. Miyaji N: Torauma no Iryojinruigaku (Medical anthropology of trauma) (in Japanese). 1st edition. Tokyo, Japan: Misuzu Shobou; 2005:77-96.

30. Toyota K, Matsumoto T: Factors relating to self-esteem in university students. (Daigakusei-no Jisonshin to Kanren-suru Shoyouin ni Kansuru Kenkyu). The Bulletin of the Institute of Human Sciences, Toyo University 2004, $1: 38-54$

doi:10.1186/1756-0500-7-924

Cite this article as: Omiya et al:: Disclosure of congenital cleft lip and palate to Japanese patients: reported patient experiences and relationship to self-esteem. BMC Research Notes 2014 7:924.

\section{Submit your next manuscript to BioMed Central and take full advantage of:}

- Convenient online submission

- Thorough peer review

- No space constraints or color figure charges

- Immediate publication on acceptance

- Inclusion in PubMed, CAS, Scopus and Google Scholar

- Research which is freely available for redistribution 